This report was prepared as an account of work sponsored by an agency of the United States Government. Neither the United States Government nor any agency thereof, nor any of their employees, makes any warranty, express or implied, or assumes any legal liability or responsibility for the accuracy, completeness, or usefulness of any information, apparatus, product, or process disclosed, or represents that its use would not infringe privately owned rights. Reference herein to any specific commercial product, process, or service by trade name, trademark, manufacturer, or otherwise does not necessarily constitute or imply its endorsement, recommendation, or favoring by the United States Government or any agency thereof. The views and opinions of authors expressed herein do not necessarily state or reflect those of the United States Government or any agency thereof.

\title{
LISTING OF UNITED STATES COMPANIES THAT SUPPLY GOODS AND SERVICES FOR GEOTHERMAL EXPLORERS, DEVELOPERS AND PRODUCERS INTERNATIONALLY
}

$\mathrm{DOE} / \mathrm{SF} / 12326--\mathrm{Tl}$

DE88 000287

August 1987

\author{
Sponsored By The \\ UNITED STATES DEPARTMENT OF ENERGY \\ Under Contract Number DE-AH03-865SF 12326
}

Developed By The

GEOTHERMAL RESOURCES COUNCIL

P.O. Box 1350

Davis, California 95617, U.S.A.

Phone: (916) 758-2360

Telex: 882410 


\section{DISCLAIMER}

This report was prepared as an account of work sponsored by an agency of the United States Government. Neither the United States Government nor any agency Thereof, nor any of their employees, makes any warranty, express or implied, or assumes any legal liability or responsibility for the accuracy, completeness, or usefulness of any information, apparatus, product, or process disclosed, or represents that its use would not infringe privately owned rights. Reference herein to any specific commercial product, process, or service by trade name, trademark, manufacturer, or otherwise does not necessarily constitute or imply its endorsement, recommendation, or favoring by the United States Government or any agency thereof. The views and opinions of authors expressed herein do not necessarily state or reflect those of the United States Government or any agency thereof. 


\section{DISCLAIMER}

Portions of this document may be illegible in electronic image products. Images are produced from the best available original document. 
NOTICE

This report was prepared as an account of work sponsored by the United States Government. Neither the United States, the United States Department of Energy, any of their employees, nor the Geothermal Resources Council, makes any warranty, express or implied, or assumes any legal liability or responsibility for the accuracy, completeness, or usefulness of any information, a pparatus, product, or process disclosed, or represents that its use would not infringe privately owned rights. Reference herein to any specific commercial product, process, or service by trade name, mark, manufacturer, or otherwise, does not necessarily constitute or imply its endorsement, recommendation, or favoring by the United States Government, any agency thereof or the Geothermal Resources Council. 


\section{GENERAL INFORMATION}

\section{The List}

This List was compiled by the Geothermal Resources Council, under a contract with the U.S. Department of Energy (DOE) during the first half of 1987. It is composed solely of U.S. companies with major offices within the United States.

All of the companies listed are involved in selling geothermally related goods and services internationally, or have the proven capability to do so. Each specific listing includes the company name, the name or title of the key contact person, address, telephone and if available a facsimile machine or telex number.

All of the venders completed a questionnaire and most were personally contacted concerning their area of expertise. The few vendors that did not contact the Council in time to be included are listed in the supplemental list with a brief description of their products. Their names do not appear in the matrices.

\section{The Matrices}

The sets of matrices were developed after careful study of the full range of geothermal operations in exploration, development and production in both direct-use (non-electric) and electrical production.

\section{How To Use The Matrices}

To determine the name of a specific vendor selling the goods or services follow this procedure:

1. Determine if what you need is sold by a supplier of goods or a supplier of services. Sometimes a single firm will do both.

2. Find the specific matrix under goods or services which indicates that particular discipline or product you are interested in.

3. Find the exact service or item in the matrix heading, move down the column under the service or item until you encounter a black dot.

4. Having located a black dot in the column, move laterally to the left to find the name of the company who supplies the service or particular item.

For example, if one is seeking the name of a company that takes infrared photographs, start with the services matrices. Find the matrix for exploration, and the subcategory for infrared photographs. By moving down the column the first black dot encountered is opposite the name of Cascadia Pacific Corporation. If you wish to continue, you may contact the company directly. If there is more than one dot in the column, you may wish to contact several or all of the companies.

If you have any questions, comments, additions or deletions concerning this List, or the Matrices, contact the Geothermal Resources Council, P.O. Box 1350, Davis, CA 95617, USA. Phone (916) 758-2360 or Telex: 882410.

NOTE: Some categories in the Matrices do not contain black dots. The columns are open because no one supplies these specific goods or services. It is felt that these categories will be filled in future issues of this list. 


\section{LISTING OF UNITED STATES COMPANIES THAT SUPPLY \\ GOODS AND SERVICES FOR GEOTHERMAL EXPLORERS, DEVELOPERS AND PRODUCERS INTERNATIONALLY}

\author{
AERO-MOBILE PRODUCTIONS \\ Brad W. Dolezal or Warren F. Dolezal \\ 4251 S. Higuera St., Ste. 103 \\ San Luis Obispo, CA 93401 \\ Telex: None $\quad$ Ph.: (805) 544-3991 \\ AGLAND INVESTMENT SERVICES, INC. \\ W.P. Mott \\ 900 Larkspur Landing Cr., Ste. 240 \\ Larkspur, CA 94939 \\ Fax: (415) 461-6958 Ph.: (415) 461-5820 \\ ALBIREO LIMITED \\ Arthur L. Lange \\ 3798 Marshall St., Ste. 3 \\ Wheat Ridge, CO 80033 \\ Telex: None \\ Ph.: (303) 431-0432 \\ ALEXANDER \& ALEXANDER, INC. \\ Account Executive \\ 370 Seventeenth St., Ste. 2300 \\ Denver, CO 80202 \\ Fax: (303) 592-5575 Ph.: (303) 592-5592 \\ ALLIED CHEMICAL
Product Manager
P.O. Box $1139 R$
Morristown, NJ 07960
Fax: (201) 455-4807 Ph.: (201) 455-3945
}

AMERON FIBERGLASS PIPE DIVISION

Neal Lambly, Western Area Manager

3583 Investment Blvd., Bldg. D, Ste. 1

Hayward, CA 94545-3737

Fax: (415) 783-4766 Ph.: (415) 783-0444

AMETEK, INC.

Vice President - Marketing

Station Square Two

Paoli, PA 19301

Fax: (215) 296-3412 Ph.: (215) 647-2121

\section{ANADRILL SCHLUMBERGER \\ Roger Ross-Smith \\ 4000 Easton Drive, No. 21 \\ Bakersfield, CA 93309 \\ Telex: None \\ Ph.: (805) 325-0271}

ANATEC LABORATORIES, INC.

Robert M. Rock, Senior Engineer

435 Tesconi Circle

Santa Rosa, CA 95401

Telex: None

Ph.: (707) 526-7200

APPLEGATE ASSOCIATES, INC.

President

134 Union Blvd., Suite 615

Lakewood, $\mathrm{CO} 80228$

Telex: 62519950 Ph.: (303) 985-5237
A-Z/GRANT/DRILLEX

Vice President

2150 W. 18th, Ste. 116

Houston, TX 77008

Fax: (713) 880-0873 Ph.: (713) 880-8888

BAILEY CONTROLS COMPANY

Gary Blume

9480 Telstar Ave., Ste. 5

El Monte, CA 91731

Fax: (818) 401-1008 Ph.: (818) 579-7555

BAKER PACKERS THERMAL SYSTEMS

Robin Dutton or Ron Repine

7777 Industry Avenue

Pico Rivera, CA 90660

Fax: (213) 949-7306 Ph.: (213) 949-9598

BALDWIN ENGINEERING, INC.

Dr. Edward Miesch, Vice President

13612 Midway Road

Dallas, TX 75244

Telex: None

Ph.: (214) 980-0784

BAMFORD, ROBERT W. \& ASSOC.

President

2315-26th Ave. East

Seattle, WA 98112

Telex: None $\quad$ Ph.: (206) 329-7966

BANK OF AMERICA NT\&SA

Richard C. Dudley

555 California St., Dept. 5044

San Francisco, CA 94104

Fax: (415) 953-1873 Ph.: (415) 622-6321

BARBER-NICHOLS ENGINEERING CO.

Engineering Administrator

6325 West 55th Ave.

Arvada, CO 80002

Fax: Same as Phone Ph.: (303) 421-8111

BARRINGER GEOSERVICES, INC.

Vice President

15000 West 6th Ave., Ste. 300

Golden, CO 80401

Fax: Same as Phone Ph.: (303) 277-1687

BAWDEN DRILLINC, INC.

Vice President - Marketing \& Business

Development.

10200 Richmond Ave.

Houston, TX 77042

Fax: (713) 974-3181 Ph.: (713) 974-3131

BECHTEL NATIONAL, INC.

Manager, New Business Development P.O. Box 3965

San Francisco, CA 94119

Fax: (415) 768-9038 Ph.:(415) 768-6108
BEN HOLT COMPANY, THE

Clement Giles

201 South Lake Avenue

Pasadena, CA 90274

Fax: (818) 584-9210 Ph.: (213) 684-2541

BERKELEY GROUP INC. (BGI,BGT,BGS)

President

1330 Broadway, Suite 1450

Oakland, CA 94612

Telex: 9103333850 Ph.: (415) 465-1744

BIG CHIEF DRILLING CO.

President

P.O. Box 14837

Oklahoma City, OK 73112

Telex: $\mathbf{7 4 7 1 5 0}$

Ph.: (405) 843-5721

BILL'S CASING TONG SERVICE, INC.

Vice Pres.-Treasurer

P.O. Box 1723

Marysville, CA 95901-1723

Telex: None

Ph.: (916) 743-5424

BIRD MACHINE CO.

John Prysi, Sales Engineer

24050 Madison St., Ste. $100 E$

Torrance, CA 90505

Fax: (213) 373-9272 Ph.: (213) 373-7622

\section{CASCADIA PACIFIC CORPORATION}

Richard J. Miller, President

Eugene V. Ciancanelli, Sec./Treas.

3358 Apostol Road

Escondido, CA 92025

Telex: None

Ph.: (619) 489-0969

CELESTIAL SOFTWARE, INC.

Ken Tashiro, Sales Manager

125 University Ave.

Berkeley, CA 94710

Telex: 556459

Ph.: (415) 420-0300

CHRISTENSEN MINING PRODUCTS

Bryan Edwards, Project Manager

4446 West 1730 South

P.O. Box 30777

Salt Lake City, UT 84130

Fax: (801) 972-6769 Ph.: (801) 974-5544

COLORADO SCHOOL OF MINES

Head Dept. Chem. \& Geochemistry

Colorado School of Mines

Golden, CO 80401

Fax: (303) 273-3283 Ph.: (303) 273-3610

\section{COLUMBIA GEOSCIENCE}

Al Waibel or David Blackwell

22495 NW Quatama Road

Hillsborough, OR 97124

Telex: None Ph.: (503) 640-9877 
COMAP EXPLORATION SERVICES, INC.

Gregory Bryan

85 South Union, \#G-155

Lakewood, CO 80228

Fax: (303) 969-9926 Ph.: (303) 969-9922

COOPERHEAT, INC.

Marketing Supervisor

4079 Ingot Street

Fremont, CA 94538

Fax: Same as Phone Ph.: (415) 490-5454

COOPER INDUSTRIES, INC./WKM DIVISION

Ray Voelkel

P.O. Box 2117

Houston, TX 77001

Fax: (713) 499-5211 Ph.: (713) 499-8511

CORRICK INTERNATIONAL

John Corrick, President

P.O. Box 66931

Scotts Valley, CA 95066

Fax: (408) 438-7367 Ph.: (408) 438-2514

COUNSIL, JOHN R., Ph.D.

Engineering Consultant

1148 Shadyoak PI.

Santa Rosa, CA 95404

Telex: None

Ph.: (707) 538-2288

CRADDOCK ENGINEERING, INC.

Ray Miedecke, Mgr.; Bus.; Devel.

18301 Irvine Blvd.

Tustin, CA 92680

Telex: 655416/188139 Ph.: (714) 730-1899

\section{CRUCIBLE COMPACTION METALS \\ Sales Eng., V.P. Sales \\ Rd. \#1 McKee \& Robb Hill Rd \\ Oakdale, PA 15071 \\ Telex: 703983 \\ Ph.: (412) 923-2670 \\ DIABLO EXECUTIVE GROUP, INC. \\ A.R. (Tony) Amor \\ P.O. Box 546 \\ Diablo, CA 94528 \\ Fax: (415) 831-9046 Ph.: (415) 831-0110}

\section{DOUGLAS ENERGY COMPANY}

Manager of Marketing

181 W. Orangethorpe Ave., Ste. D

Placentia, CA 92670

Fax: (714) $5243341 \quad$ Ph.: (714) 524-3338

DOW CHEMICAL USA

Market Manager

P.O. Box 3387

Houston, TX 77253

Fax: (713) 978-2749 Ph.: (713) 978-3893

DRAVO CONSTRUCTORS, INC.

G.D. Stewart, Sales Manager

226 Airport Parkway, Ste. 550

San Jose, CA 95110

Fax: (408) 298-2107 Ph.: (408) 291-2853

DREsSER atlas

G.C. Calongne - Far East Area Mgr.

P.O. Box 1407

Houston, TX 77251

Fax: (713) 972-4862 Ph.: (713) 972-6529
DRILLING RESOURCES DEVEL. CORP.

Manager, Drilling Services

6111 E. Skelly Dr., Ste. 415

Tulsa, OK 74135

Telex: 4909964133

Ph.: (918) 664-9010

\section{EARTHQUEST}

President - Geologist

2161 Shattuck Ave., Ste. 210

Berkeley, CA 94704

Telex: None

Ph.: (415) 845-3800

EASTMAN CHRISTENSEN CO.

Nic Nickels, Base Manager

320 Tesconi Circle, Unit P

Santa Rosa, CA 95401

Fax: (707) 523-1398 Ph.: (707) 523-1751

EATON INDUSTRIES OF HOUSTON, INC.

V.P. Engineering

1980 Post Oak Blvd., Ste. 2000

Houston, TX 77056

Fax: (713) 627-8985 Ph.: (713) 627-9764

EG\&G CHANDLER ENGINEERING

Marketing Executive

7707 E. 38th Street

Tulsa, OK 74145

Fax: (918) 663-5619 Ph.: (918) 627-1740

EIMCO PROCESS EQUIPMENT CO.

Robert W. Moore, Jr., District Mgr.

P.O. Box 5485

Garden Grove, CA 92645-0485

Fax: (714) 895-5014 Ph.: (714) 898-5561

ELECTROMAGNETIC SURVEYS, INC.

President

2161 Shattuck Ave., Ste. 317

Berkeley, CA 94704

Telex: None

Ph.: (415) 845-8745

\section{ELLIOTT COMPANY}

Mgr., Engineered Products

P.O. Box 384

City of Industry, CA 91749-0384

Fax: (818) 964-0506 Ph.: (818) 810-2121

ENERGY AND NATURAL RESOURCES CONSULTANTS, INC.

President

641 Strings

San Antonio, TX 78216

Telex: None

Ph.: (512) 341-3489

\section{ENERGYLOG CORPORATION}

Randy Palmer, Operations Mgr.

P.O. Box 162580

Sacramento, CA 95816

Telex: None Ph.: (916) 452-7541

ERSHIGS, INC

Regional Sales Manager

742 Marine Drive

Bellingham, WA 98225

Fax: Same as Phone Ph.: (206) 733-2620

\section{EXLOG/SMITH (A)}

Vice President, Gen. Mgr.

75-161 Sego Lane, Ste. F-16

Palm Desert, CA 92260

Telex: None

Ph.: (619) 346-8889
EXIOG/SMITH Geothermal (B)

Senior Operations Mgr.

P.O. Box 1349

195 Dry Creek Rd., Bldg. 1A

Healdsburg, CA 95448

Telex: None

Ph.: (707) 431-1700

\section{EXPLORATION GEOTHERMICS}

Principal Geophysicist

4636 Mission George Pl., Ste. 200

San Diego, CA 92120

Telex: None Ph.: (619) 582-4325

FOSTER/W.L.G. SYSTEMS, DIVISION

Bill Godare

3600-C Standish Avenue

Santa Rosa, CA 95407

Telex: $384556 \quad$ Ph.: (707) 585-1981

FRANCIS, RICHARD N.

Consultant

P.O. Box 9028

Reno, NV 89507

Telex: None

Ph.: (702) 322-5949

GASO PUMPS, A Goulds Pump Co.

Marketing Manager

P.O. Box 2069

Tulsa, OK 74101

Fax: (918) 446-4904 Ph.: (918) 446-4551

GEARHART MWD

General Manager

2700 Post Oak Blvd., Ste. 19

Houston, TX 77056

Fax: (713) 871-6490 Ph.: (713) 871-6519

\section{GEOHEAT CENTER}

Paul Lienau, OR Inst. of Tech.

3201 Campus Drive

Klamath Falls, OR 97601

Telex: None

Ph.: (503) 882-6321

\section{GEOPHYSICAL RESEARCH CORP.}

Manager Sales

P.O. Box 581570

Tulsa, OK 74158-1570

Fax: (918) 838-8846 Ph.: (918) 834-9600

GEOPHYSICAL SYSTEMS CORP.

President

2085 East Foothill Blvd.

Pasadena, CA 91107

Fax: (818) 795-4536 Ph.: (818) 793-9511

GEOPHYSICS GROUP, THE

Manager of Operations

3798 Marshall Street

Denver, CO 80033

Telex: None

Ph.: (303) 431-0432

\section{GEOTECH DATA}

Barry Williams

13730 Adrian Street

Poway, CA 92064

Telex: None

Ph.: (619) 748-8341

GEOTHERMAL DEVELOPMENT ASSOCS.

President

251 Ralston St.

Reno, NV 89503

Telex: None

Ph.: (702) 322-0938 
GEOTHERMAL POWER COMPANY

Gary Shulman

1460 W. Water Street

Elmira, NY 14905

Telex: None

Ph.: (607) 733-1027

GEOTHERMAL RESOURCES COUNCIL

David N. Anderson

P.O. Box 1350

Davis, CA $95617-1350$

Telex: 882410

Ph.: (916) 758-2360

\section{GEOTHERMAL WORLD CORP.}

Editor

17304 Village 17

Camarillo, CA 93010

Telex: None

Ph.: (805) 482-3068

GEOTHERMEX, INC.

James B. Koenig, President

5221 Central Ave., Ste. 201

Richmond, CA 94804

Fax: (415) 527-8164 Ph.: (415) 527-9876

GLOBAL GEOCHEMISTRY CORP.

I.R. Kaplan

6919 Eton Ave.

Canoga Park, CA 91303

Fax: (818) 992-8940 Ph.: (818) 992-4103

\section{GRACE DRILLING}

Pat Sullivan

6804 Fishback

Bakersfield, CA 93308

Fax: (805) 589-5115 Ph.: (805) 589-6581

GRAHAM MANUFACTURING CO., INC. Executive Vice President

20 Florence Avenue

Batavia, NY 14020

Fax: (716) 343-1097 Ph.: (716) 343-2216

GRAYSTAR TECHNICAL SERVICES

President

P.O. Box 100506

Anchorage, AK 99510-0506

Telex: 20026560

Ph.: (907) 243-5661

GRACE, SHURSEN, MOORE \& ASSOC.

Manager

P.O. Box 9920

Amarillo, TX 79105

Telex: 910-898-4121 Ph.: (806) 358-6894

HALLIBURTON SERVICES

Cementing Services Coordinator

P.O. Drawer 1431

Duncan, OK 73536-0206

Fax: (405) 251-3227 Ph.: (405) 251-3760

HARLEY MURRAY, INC.

Dispatcher or Sales

1754 E. Mariposa Rd.

Stockton, CA 95205

Fax: (209) 466-0550 Ph.: (209) 466-0266

HAYMOND AND ASSOCIATES

President

4711 South Fairplay Court

Denver, CO 80015-2104

Telex: None

Ph.: (303) 680-9122
HI-TECH FLUIDS, INC.

Peter Gallus

980 Hilda Street

Anaheim, CA 92806

Telex: None

Ph.: (714) 772-6657

HYDRIL COMPANY

District Manager

P.O. Box 60458

Houston, TX 77205-0458

Telex: 27-5114 Ph.: (713) 449-2000

INSULATED WIRE, INC.

Arthur Nixon

P.O. Box 37

Ronkonkoma, NY 11779

Fax: (516) 588-0074 Ph.: (516) 981-7424

INTERMOUNTAIN TECH'S., INC.

Vice President

P.O. Box 1604

Idaho Falls, ID 83403-1604

Fax: Same as Phone Ph.: (208) 523-7255

\section{INTER-TRADE SUPPIY}

President

1205 Gravenstein Hywy. South

Sebastopol, CA 95472

Telex: 4930733

Ph.: (707) 823-8642

JACOBSON, IIMMY CONSULTANT

Jimmy Jacobson

11009 W. 65 Way

Arvada, CO 80004

Telex: 450286

Ph.: (303) 420-8523

JAMES GEO ASSOCIATES.

Laurence $P$. James

P.O. Box 226

Golden, $\mathrm{CO} 80402$

Telex: None

Ph.: (303) 279-0493

JEROME INSTRUMENT CORP.

Director of Sales \& Marketing

P.O. Box 336

Jerome, AZ 86331

Fax: (602) 634-9304 Ph.: (602) 634-4263

JOHNSON CONTROLS, INC.

James Haas

2110 So. Anne Street

Santa Ana, CA 92799-5129

Telex: $9103803401 \quad$ Ph.: (714) 754-5767

JOHNSTON PUMP COMPANY

Manager, Geothermal Div.

16021 Aclolante St.

Azusa, CA 91702

Telex: $67-0316$

Ph.: (818) 969-5927

IORDAN, APOSTAL, RITTER ASSO., INC.

President

Adminstration Building 7

Davisville, RI 02854

Telex: None

Ph.: (401) 884-3014

KENNEDY \& ASSOCIATES

John Kennedy

21241 Ventura Blvd.

Woodland Hills, CA 91364

Telex: 361674

Ph.: (818) 704-0711

KINEMETRICS, INC.

John G. Diehl, Mgr. Sales \& Services

222 Vista Avenue

Pasadena, CA 91107

Fax: (818) 795-0868 Ph.: (818) 795-2220
KINETICS TFCHNOLOGY INT'L CORP.

Process Sales Engineer

1333 So. Mayflower Avenue

Monrovia, CA 91016

Fax: (818) 447-4009 Ph.: (818) 303-4711

KUSTER COMPANY

John Jacobsen, Manufacturing Eng. P.O. Box 7038

Long Beach, CA 90807

Fax: (213) 426-7897 Ph.: (213) 595-0661

LITTE, ARTHUR D. VALUATION

John D. Glenn

P.O. Box 4028

Woodland Hills, CA 91365-4028

Telex: 678428

Ph.: (818) 702-0400

LOFFLAND BROTHERS COMPANY

Exec. V.P., International Operations

P.O. Box 2847

Tulsa, OK 74101-2847

Fax: (918) 664-0828 Ph.: (918) 622-9330

MAFI-TRENCH CORPORATION

Vice President

3037 Industrial Parkway

Santa Maria, CA 93455

Fax: (805) 925-3861 Ph.: (805) 928-5757

MARLEY COOLING TOWER CO.

V.P. International Division

5800 Foxridge Drive

Mission, KS 66202

Fax: (913) 362-3610 Ph.: (913) 362-1818

MARS ASSOCIATES, INC.

President

3644 E. McDowell Rd., \#207

Phoenix, AZ 85008

Telex: 668466

Ph.: (602) 267-8008

MCDANIEL, SCOTT

Consulting Geologist

975 Melba Drive

Reno, NV 89503

Telex: None

Ph.: (702) 323-8224

McMURDIE, DENNIS \& ASSOC.

President

9430 Research \#400

Austin, TX 78759

Telex: 650-2637011 Ph.: (512) 343-4587

MESQUITE GROUP, INC.

Don A. Campbell

P.O. Box 1283

Fullerton, CA 92632

Telex: None

Ph.: (714) 738-8224

MICHELS, DON ASSOCIATES

Don Michels

P.O. Box 895

Whittier, CA 90608

Telex: None

Ph.: (213) 698-5728

\section{MICROGEOPHYSICS CORPORATION}

David Butler

10900 W. 44th Avenue

Wheat Ridge, CO 80033

Telex: None Ph.: (303) 424-0499

MIDWAY FISHING TOOL CO.

F. Desta Jones, President

3501 Chester Avenue

Bakersfield, CA 93301

Telex: None

Ph.: (805) 327-4471 
MISSION POWER ENGINEERING CO.

President

38 Executive Park

Irvine, CA 92714

Fax: (714) 756-3855 Ph.: (714) 756-3800

MONTCOMERY DRILLING COMPANY
Rob Montgomery
P.O. Box 80307
Bakersfield, CA 93380
Telex: None Ph.: (805) 393-9620

MORRISON-KNUDSEN ENGINEERS

Hydrology/Reservoir Eng. Mgr.

P.O. Box 79

Boise, ID 83707

Fax: (208) 386-7186 Ph.: (208) 386-5000

NASH ENGINEERING

Paul Bernard

310 Wilson Avenue

Norwalk, CT 06856

Telex: None

Ph.: (203) 852-3637

NASH-KINEMA, INC.

V.P., Engineering

P.O. Box 176

700 Elizabeth-Glassport Rd

Elizabeth, PA 15037

Fax: (412) 384-4880 Ph.: (412) 384-3610

NATIONAL SUPPLY CO.

Floyd King

P.O. Box 2245

Bakersfield, CA 93303

Telex: None Ph.: (805) 589-5750

NATURAL RESOURCES LAB., INC.

Ron Keil

1100 Simms Street

Golden, CO 80401

Telex: None

Ph.: (303) 233-8155

NL SHAFFER

Anthony Onorato

200 North Berry

Brea, CA 92621

Telex: 678475

Ph.: (714) 671-4046

OTIS ENGINEERING CORP.

Russell Bayh

P.O. Box 819052

Dallas, $T \times 75381$

Telex: 0732390

Ph.: (214) 323-3694

PEERLESS MANUFACTURING CO
Sales Manager
P.O. Box 20657
Dallas, TX 75220
Telex: 073-2345 Ph.: (214) 357-6181
PETREX
Sales - Environmental
605 Parfet, Suite 100
Lakewood, CO 80215
Fax: (303) 238-2522 Ph.: (303) 238-0090
PETROCHEM INSULATION, INC.
Business Devel. Mgr.
110 Corporate Place
Vallejo, CA 94590
Fax: (707) 644-4908 Ph.: (707) 644-7455

PIPE RESTORATION SYSTEMS, INC.

James G. Dodd

25 Business Park Drive

Bamford, CT 06405

Fax: (203) 481-2883Ph.: (203) 488-5955

PIPE SALES COMPANY

Troy B. Allison, Mgr. of Sales

5401 Business Park So., Ste. 206

Bakersfield, CA 93309

Telex: None

Ph.: (805) 323-2031

PRUETT INDUSTRIES, INT'L.

Edward Pruett

8915 Rosedale Highway

Bakersfield, CA 93312

Telex: $4992440 \quad$ Ph.: (805) 589-2768

RADIAN CORPORATION

Geothermal Program Mgr.

P.O. Box 9948

Austin, TX 78766

Fax: (512) 454-7129 Ph.: (512) 454-4797

REED TOOL COMPANY

Don Wilson

P.O. Box 2119

Houston, TX 77252

Telex: $4620579 \quad$ Ph.: (713) 924-5238

ROHRBACK COSASCO SYSTEMS

Sales Manager

$11841 \mathrm{E}$. Smith Ave.

Santa Fe Springs, CA 90670

Fax: (213) 949-3065 Ph.: (213) 949-0123

\section{ROTOFLOW CORPORATION}

Homer Fager

540 E. Rosecrans Ave.

Gardena, CA 90248

Fax: (213) 329-8502 Ph.: (213) 329-8447

SAI ENGINEERS, INC.

President

3030 Patrick Henry Drive

Santa Clara, CA 95054-0979

Fax: (408) $727-4733 \quad$ Ph.: (408) 727-6328

SCHNEIDER, J.R. Co., Inc.

Gene Pecci

849 Jackson St.

Benicia, CA 94510

Telex: 34-0545 Ph.: (707) 745-0404

SCIENTIFIC DRILLING INTERNATIONAL

V.P., Operations

14715 Jersey Shore Dr.

Houston, TX 77047

Fax: (713) 433-7166 Ph.: (713) 433-0010

S-CUBED

Mgr., Resources Technology

3398 Carmel Min. Rd.

San Diego, CA 92121-1095

Fax: (619) 755-0474 Ph.: (619) 453-0060

SOUTHPORT EQUIPMENT CORP.

6914 Canby Ave., Suite 102

P.O. Box 758

Reseda, CA 91335

Fax: (818) 705-8762 Ph.: (818) 705-8300
SOUTHWEST RESEARCH INSTITUTE

Director, Dept. of Geoscience

P.O. Box 28510

San Antonio, TX 78284

Fax: (512) 522-3396 Ph.: (512) 522-2715

S.P.S., INC.

Victor S. Warminger, Dir. Sales

P.O. Box 380006

Miami, FL 33138

Fax: (305) 264-9287

Ph.: (305) 754-7766

STONE \& WEBSTER ENG. CO.

Geothermal Bus. Devel. Mgr.

245 Summer Street

Boston, MA 02107

Fax: (617) 589-2156 Ph.: (617) 589-6627

SYLVESTER ASSOCIATES

President

P.O. Box 1327

Santa Rosa, CA 95402

Fax: (707) 576-0437 Ph.: (707) 523-3228

SYSTEMS APPLICATION ENG., INC.

President

3655 Westcenter Drive

Houston, TX 77042

Fax: (713) 783-3434 Ph.: (713) 783-6020

TAM INTERNATIONAL

L. Bentley Sanford/Doc Stokley

4620 Southerland

Houston, TX 77092

Telex: 9108811659 Ph.: (713) 462-7617

TAYLOR FORGE ENGINEERED

SYSTEMS, INC.

Mgr of Contract Administration

First and Iron Streets

Paola, KS 66071

Fax: Same as Phone Ph.: (913) 294-5331

TERRASENSE, INC.

Interpretation Manager

P.O. Box 3651

Sunnyvale, CA 94086

Telex: 357-435 Ph.: (408) 745-0713

TETRA RECOVERY SYSTEMS

Arthur Geier, Western Mgr.

P.O. Box 3849

Santa Rosa, CA 95402

Fax: (707) 523-1029 Ph.: (707) 523-2803

THERMASOURCE, INC.

Louis E. Capuano Jr., President

P.O. Box 1236

Santa Rosa, CA 95402

Fax: (707) 523-1029 Ph.: (707) 523-2960

THERMOCHEM, INC.

Jean Stewart

6119 Old Redwood Hywy, Ste. A-2

Santa Rosa, CA 95401

Telex: None

Ph.: (707) 575-1310

TONTO DRILLING SERVICES

George McLaren

2701 West 900 South

Salt Lake City, UT 84101

Fax: (801) 973-2994 Ph.: (801) 974-0645 
TRACOR ATLAS, INC.

Sales Manager

9441 Baythorne Drive

Houston, TX 77041-7709

Fax: (713) 462-1831 Ph.: (713) 462-6116

TRI-STATE OIL TOOL INDUSTRIES, INC.

Regional Engineer

P.O. Box 5757

Bossier City, LA 71171-5757

Fax: (318) 747-0652 Ph.: (318) 746-3800

TRW REDA PUMP DIVISION

Mgr. International Marketing

P.O. Box 1181

Bartlesville, OK 74005

Fax: (918) 661-2101 Ph.: (918) 661-2000

T.S.T.C.O.

Thomas S. Tortorete

1637 Meadowlark Dr.

Fairfield, CA 94533

Telex: None

Ph.: (707) 422-8418

\section{UNIVERSITY OF UTAH RESEARCH} INSTITUTE

Technical Vice President

391 Chipeta Way, Ste. C

Salt Lake City, UT 84108

Fax: (801) 524-3453 Ph.: (801) 524-3422

VALLEY ENGINEERS, INC

Tom Flynn

P.O. Box 12227

Fresno, CA 93777

Telex: None

Ph.: (209) 237-9173
VAN HOUTEN CONSULTANTS

Gary E. Van Houten, President

422 Petaluma Blvd. North, Ste. B

Petaluma, CA 94952

Telex: VH CONSULT Ph.: (707) 762-1132

VICE, DAN H. \& ASSOCIATES

Dan H. Vice, Cons. Geologist

449 Hart-Albin Bldg.

Billings, MT 59101

Telex: None

Ph.: (406) 248-3341

WATERS INTERNATIONAL INC.

Peyton D. Waters, Jr.

5615 Clara Road

Houston, TX 77041

Fax: (713) 466-5981 Ph.: (713) 466-0026

WELCH \& HOWELL DRILLING, INC.

Vice President

2453 Mandeville Canyon Rd.

Los Angeles, CA 90049

Telex: None

Ph.: (213) 472-2988

WELLTECH, INC.

Sug Roberts

410 17th St., Ste. 1375

Denver, CO 80202

Telex: None

Ph.: (303) 573-1666

WHEELER, GEORGE $H$.

Engineer/Geologist

154 Washakie

Lovell, WY 82431

Telex: None

Ph.: (307) 548-2840
WHEELER, HERBERT E., JR.

Petroleum Engineer

2050 Maple Glen Rd.

Sacramento, CA 95864

Telex: (None) Ph.: (916) 485-2715

WILLAMS TOOL CO., INC.

V.P. Marketing

P.O. Box 6155

Ft. Smith, AR 72906

Telex: 536016 Ph.: (501) 646-8866

ZENITH AERIAL, INC.

Marketing Director

P.O. Box 2710-423

Huntington Beach, CA 92647

Telex: None $\quad$ Ph.: (714) 968-8536

\section{ZONGE ENGINEERING \& RESEARCH}

ORGANIZATION, INC.

Director of Marketing

3322 E. Fort Lowell Rd.

Tucson, AZ 85716

Fax: (602) 325-1588 Ph.: (602) 327-5501

\section{VENDOR LIST SUPPLEMENT}

\author{
APPLIED POWER TECHNOLOGY \\ David Weiss \\ 3000 Sand Hill Road \\ Bldg. 3, Ste. 285 \\ Menlo Park, CA 94025 \\ Fax: (415) 854-9877 Ph.: (415) 854-9870 \\ Project management and equity \\ financing.

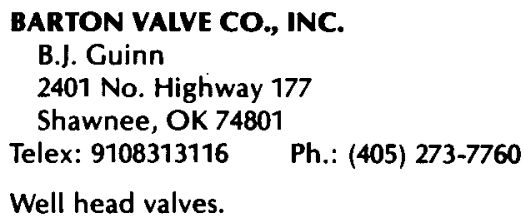

\section{BRIDWELL CONTROLS}

Barbara Bridwell

1733 Center Avenue

Martinez, CA 94553

Telex: None

Ph.: (415) 228-5084

Computer control programs for geothermal well fields and power plants.

\author{
CALFORNIA ENERGY COMMISSION \\ Energy Technology Export \\ Program Manager \\ 1516 9th Street, MS-45 \\ Sacramento, CA 95814 \\ Telex: 324-3029 \\ Ph.: (916) 324-3449
}

Renewable energy, export program and geothermal sales information.

\section{DRESSER-RAND}

Mike Beyham

20602 East 81st Street

Telex: $4637045 \quad$ Ph.: (918) 451-1550

Air drilling services.

\section{GEOTHERMAL MANAGEMENT CO.}

Gerald W. Huttrer

Box 2980

Evergreen, CO 80439

Fax: (303) 674-3820 Ph.: (303) 670-3454

Geothermal project management, exploration and economic evaluation.
Broken Arrow, OK 74014

\section{HALLIBURTON SERVICES}

Lanier Lohn

P.O. Box 10988

Bakersfield, CA 93389

Telex: 4720177

Ph.: (805) 327-0148

Well cementing services.

H\&H OIL TOOL CO., INC.

Joe Turk

1000 Church Road

Rio Vista, CA 94571

Telex: None

Ph.: (707) 374-6493

Blowout preventers, drilling tools and tubulars.

\section{HUGHES TOOL COMPANY \\ Tom Haynes \\ P.O. Box 2539 \\ Houston, TX 77252-2539}

Fax: (713) 924-2007 Ph.: (713) 924-2222

Drill bits. 
J.M. ENERGY CONSULTANTS, INC.

Jack McNamara

11752 San Vicente Blvd.

Los Angeles, CA 90049

Telex: None

Ph.: (213) 826-8269

Legal, regulatory and financial services.

\section{LONGYEAR COMPANY \\ Russel Beddow \\ 7773 West Seldon Land \\ Peoria, AZ 85345 \\ Fax: (602) 486-1885 Ph.: (602) 486-1881 \\ Temperature gradient well drilling to $2500 \mathrm{~m}$. \\ MERIDIAN CORPORATION \\ Deepak Kenkeremath \\ 4300 King Street, Ste. 400 \\ Alexandria, VA 22302 \\ Telex: $469248 \quad$ Ph.: (703) 998-3600 \\ Studies, analyses, policy and program development \\ MK FERGUSON COMPANY \\ R.M. Sullivan \\ 1 Eireview Plaza \\ Clearland, $\mathrm{OH} 44114$ \\ Telex: None \\ Ph.: (216) 523-5600 \\ Power plant design and construction.}

N.L. BAROID

Gene Polk

Box 280

Sandia Park, NM 87047

Telex: None

Ph.: (505) 281-5191

Drilling mud, additives, engineering and training.

\section{POSI-SEAL INTERNATIONAL}

Fred Riccioli

Route 49 and U.S. 95

No. Stonington, CT 06359

Telex: 247317

Ph.: (203) 599-1140

High performance valves.

\section{SMITH INTERNATIONAL}

Ken Grumbles/Judy Fenton

P.O. Box 60068

Houston, TX 77032

Fax: (713) 443-6350 Ph.: (713) 443-3376

Drilling bits and tools.

TERRA TEK INC.

Bennie DeBibona

400 Wakara Way

Salt Lake City, VT 84108

Fax: (801) 584-2406 Ph.: (801) 584-2400

Drilling research - geoscience.
THERMAL POWER CO.

William D'Olier

601 California St.

San Francisco, CA 94108

Telex: $\mathbf{3 4 3 8 7}$

Ph.: (415) 981-5700

Full range of services for international exporation and development.

\section{WELL PRODUCTION TESTING}

Robert Nicholson

P.O. Box 69

Carlsbad, CA 92008

Telex: Non

Ph.: (619) 438-7733

Drilling, reservoir engineering, reservoir testing and evaluation.

WESTEC SERVICES, INC.

Brian Boswell

5510 Morehouse Dr.

San Diego, CA 92121-1709

Fax: (619) 458-9044 Ph.: Same as Fax

Mechanical and electrical design engineering, power plant maintenance and operation, and feasibility studies and analysis.

\section{LIST OF MAJOR U.S. GEOTHERMAL DEVELOPERS WITH INTERNATIONAL OPERATIONS}

\author{
CHEVRON RESOURCES CO. \\ Gene Cole \\ P.O. Box 7147 \\ San Francisco, CA 94120 \\ Telex: $\mathbf{4 7 0 0 7 4}$ \\ Ph.: (415) 894-7700 \\ FREEPORT MCMORAN RESOURCE \\ PARTNERS \\ C.J. Von Hoene \\ P.O. Box 11279 \\ Santa Rosa, CA 95406 \\ Fax: (707) 578-2798 Ph.: (707) 578-2701
GEOTHERMAL RESOURCES INTER- NATIONAL
Jim Combs
1825 So. Grant St. Ste 900
San Mateo, CA 94402
Fax: (415) 349-4801 Ph.: (415) 349-8181

\section{KENNECOTT EXPLORATIONS}

(Australia) Ltd.

Larry Grogan

P.O. Box 278

Imperial, CA 92251

Fax: (619) 355-4253 Ph.: (619) 355-2427

\section{OXBOW GEOTHERMAL CORP.}

Douglass Powell

200 So. Virginia St.

Reno, NV 89501

Telex: None

Ph.: (702) 322-1300

THERMAL POWER CO.

William D'Olier

601 California St.

San Francisco, CA 94108

Telex: 34387

Ph.: (415) 981-5700
UNOCAL, GEOTHERMAL DIV.

Chester F. Budd

1201 West 5th Street

P.O. Box 7600

Los Angeles, CA 90051

Telex: 67-7593

Ph.: (213) 977-7694 
Matrices of GOODS 


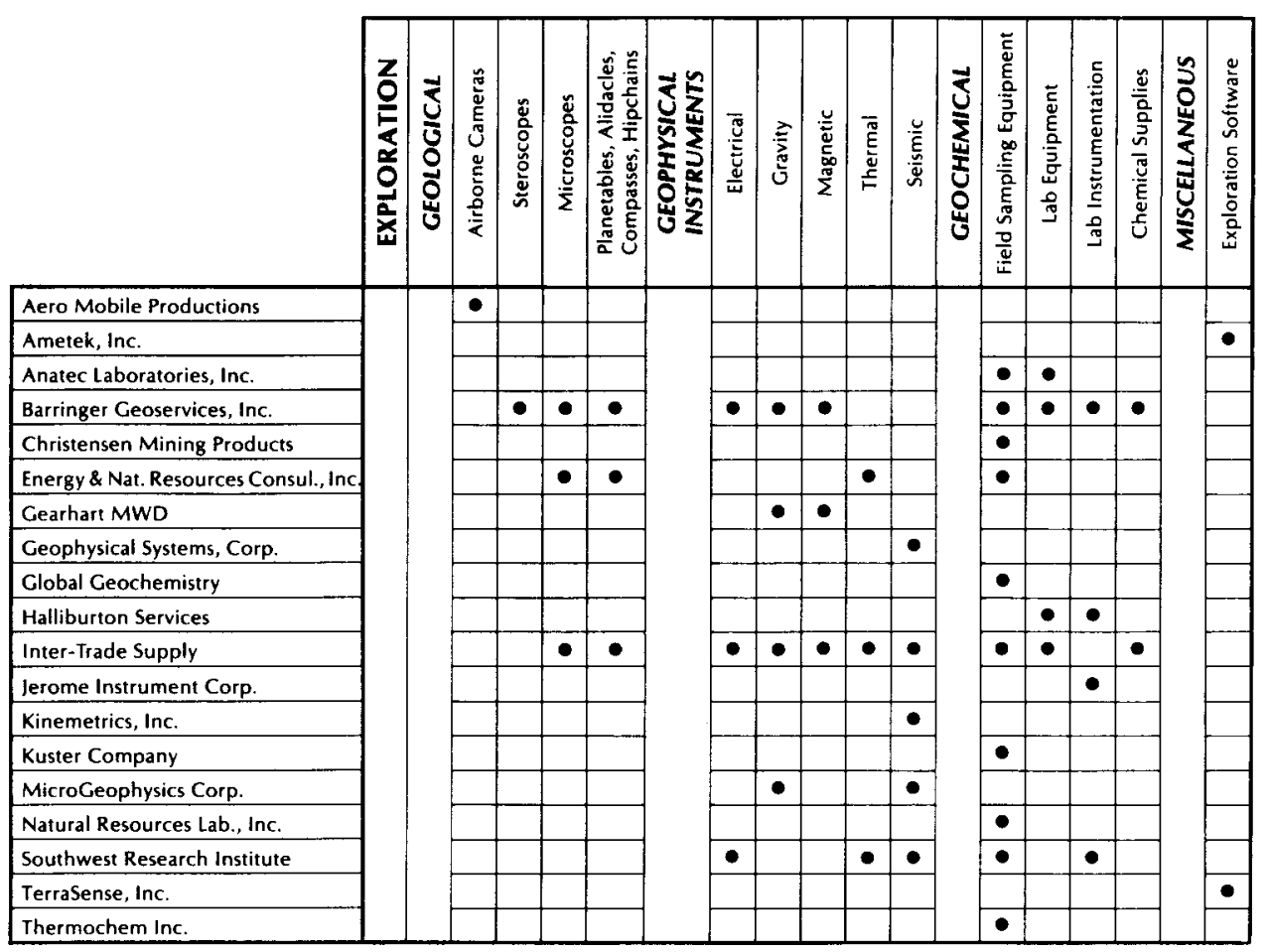




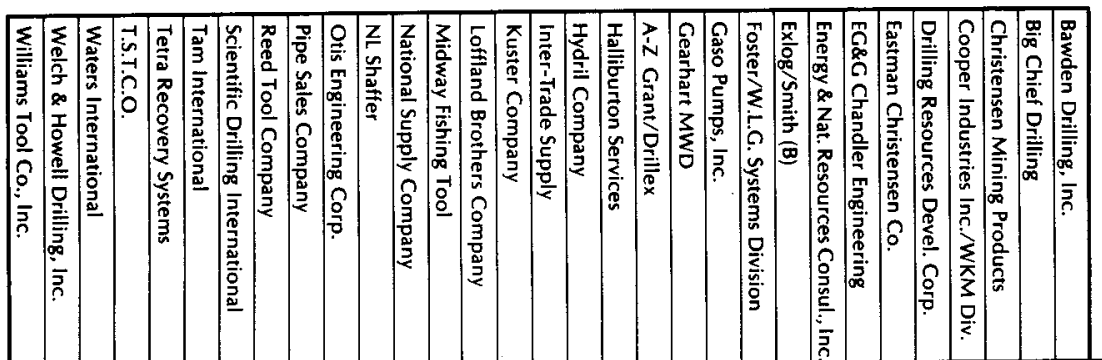

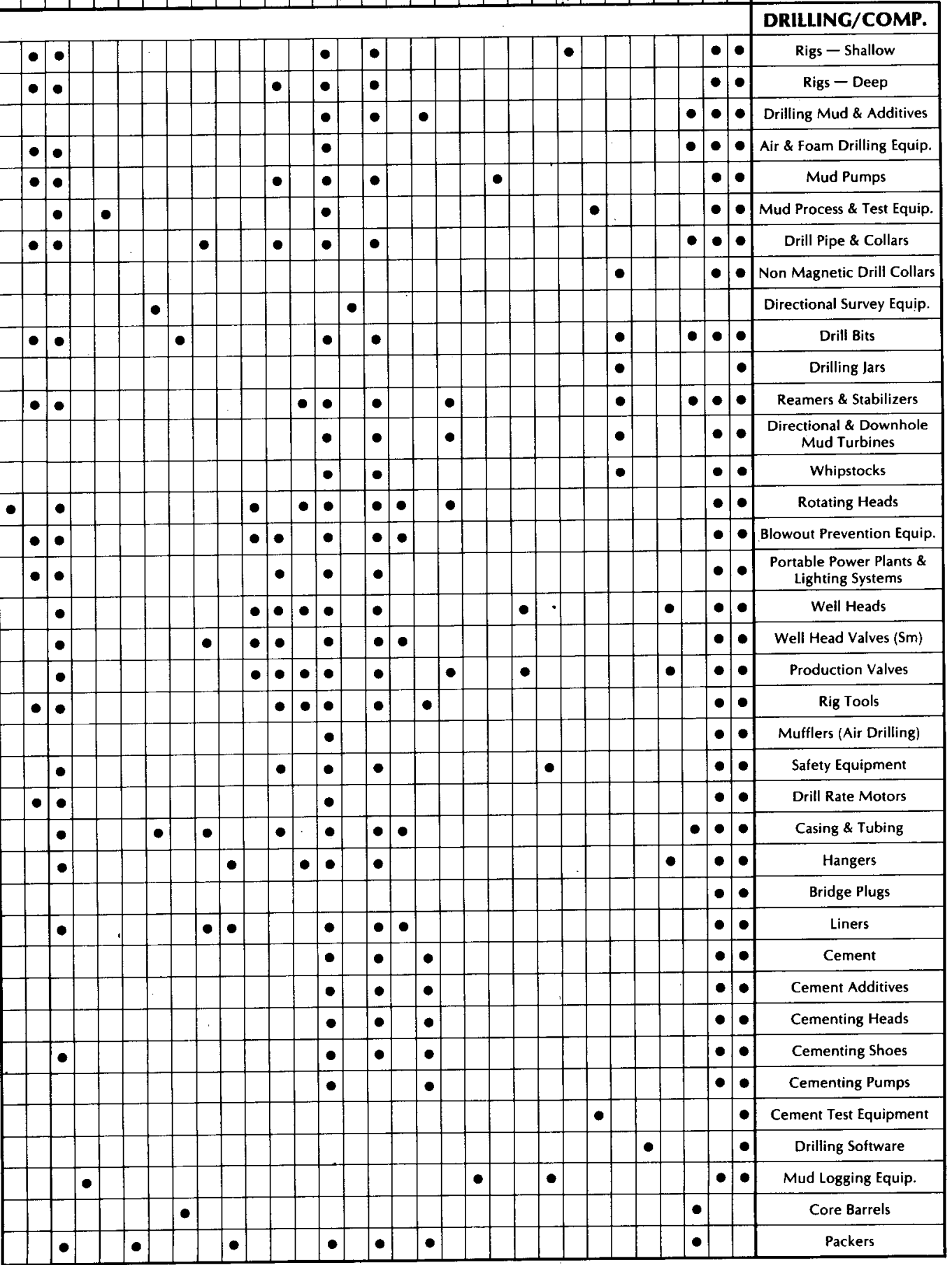



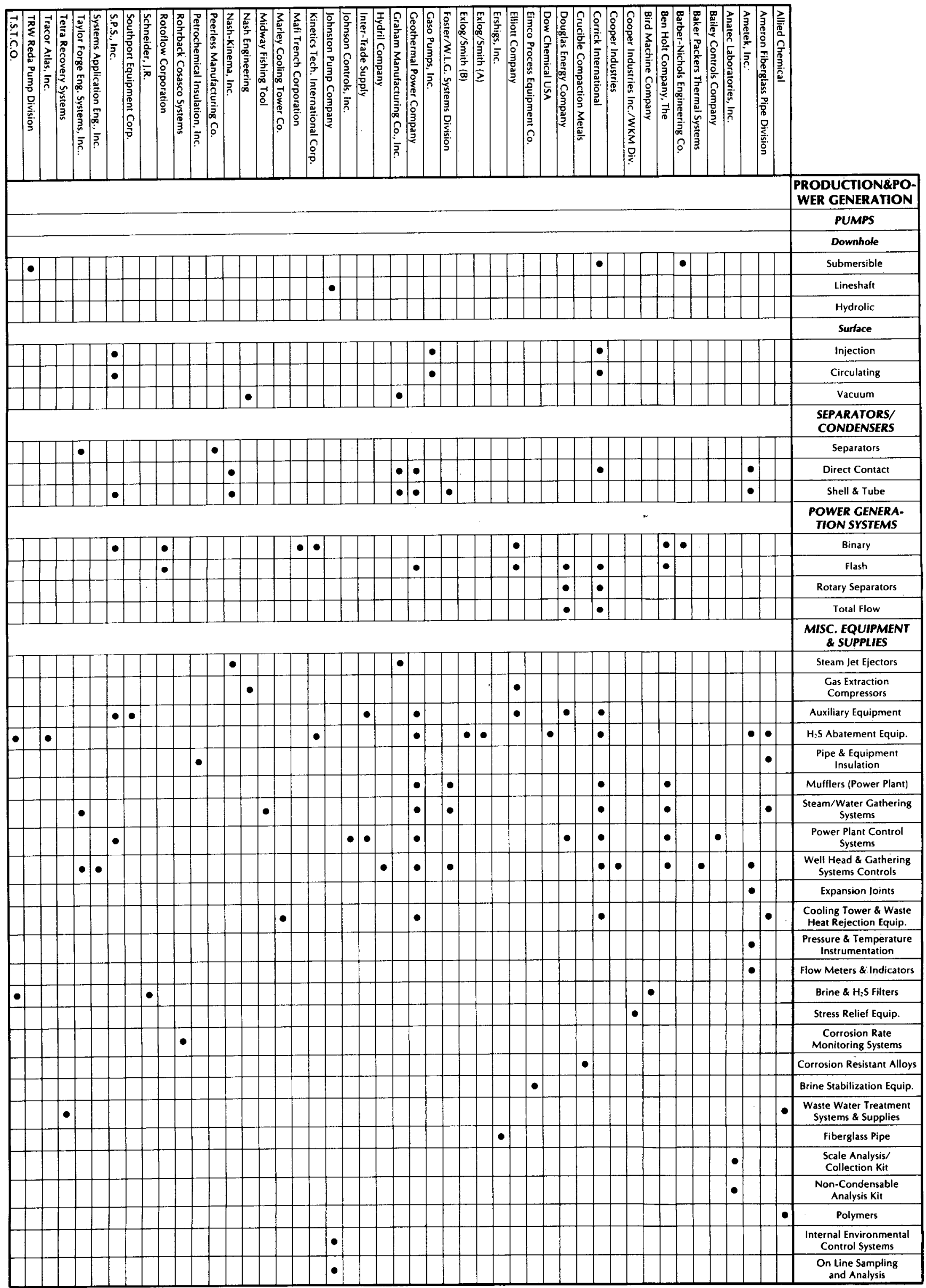


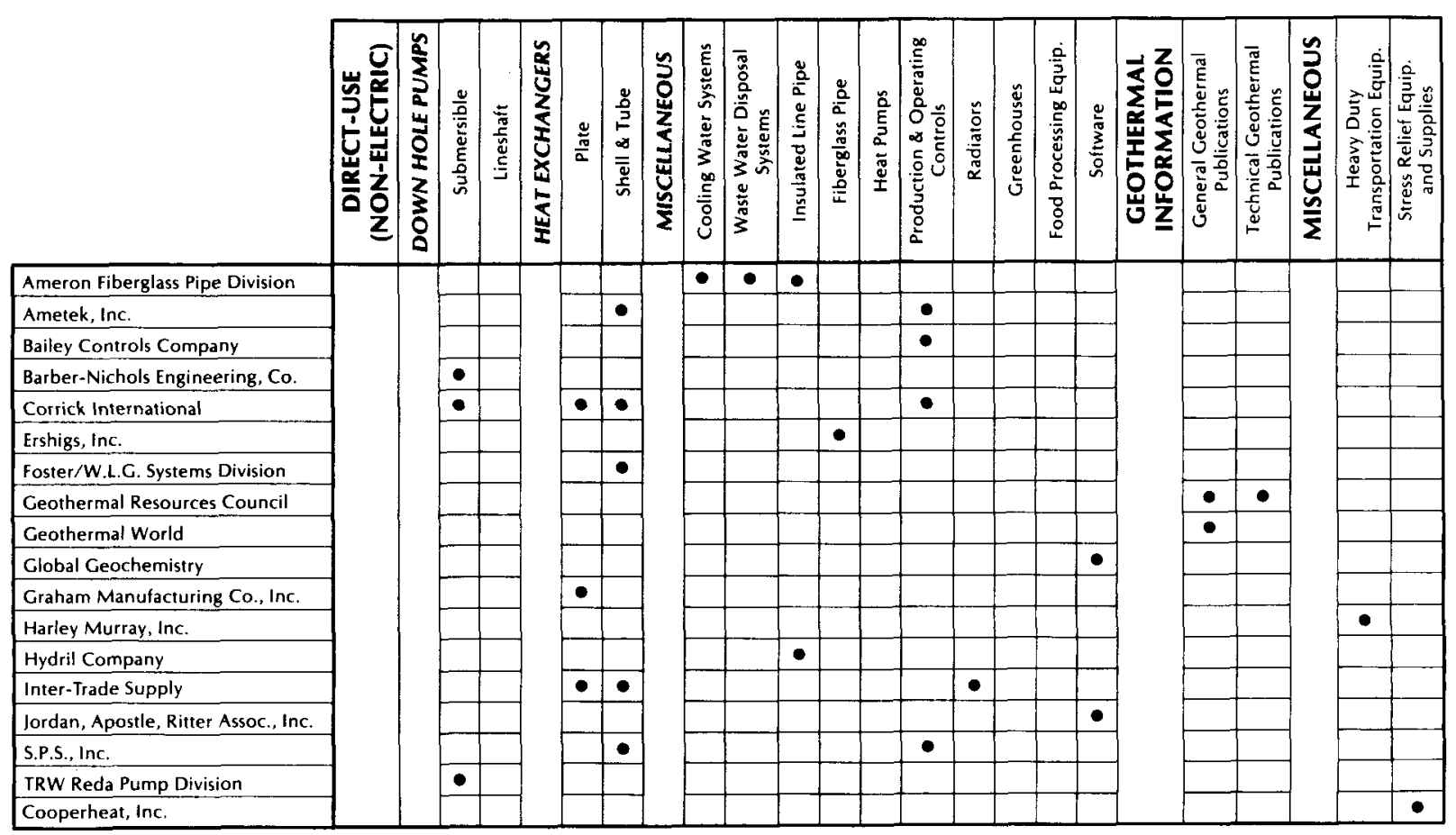



Matrices of SERVICES 
EXPLORATION CONT.

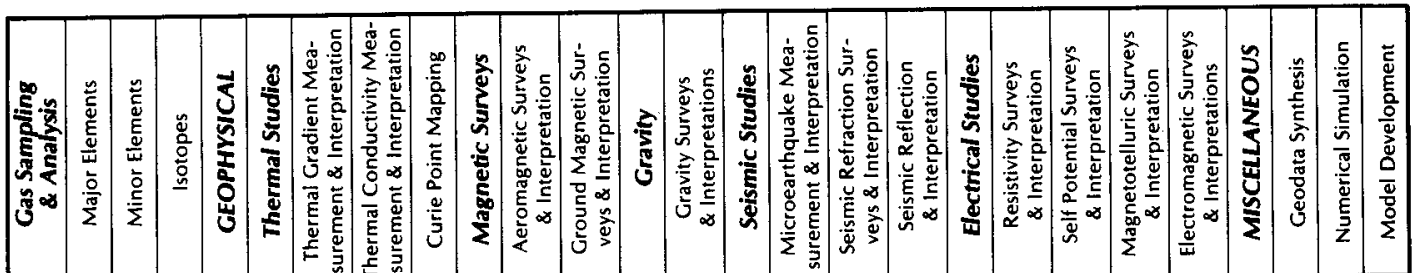

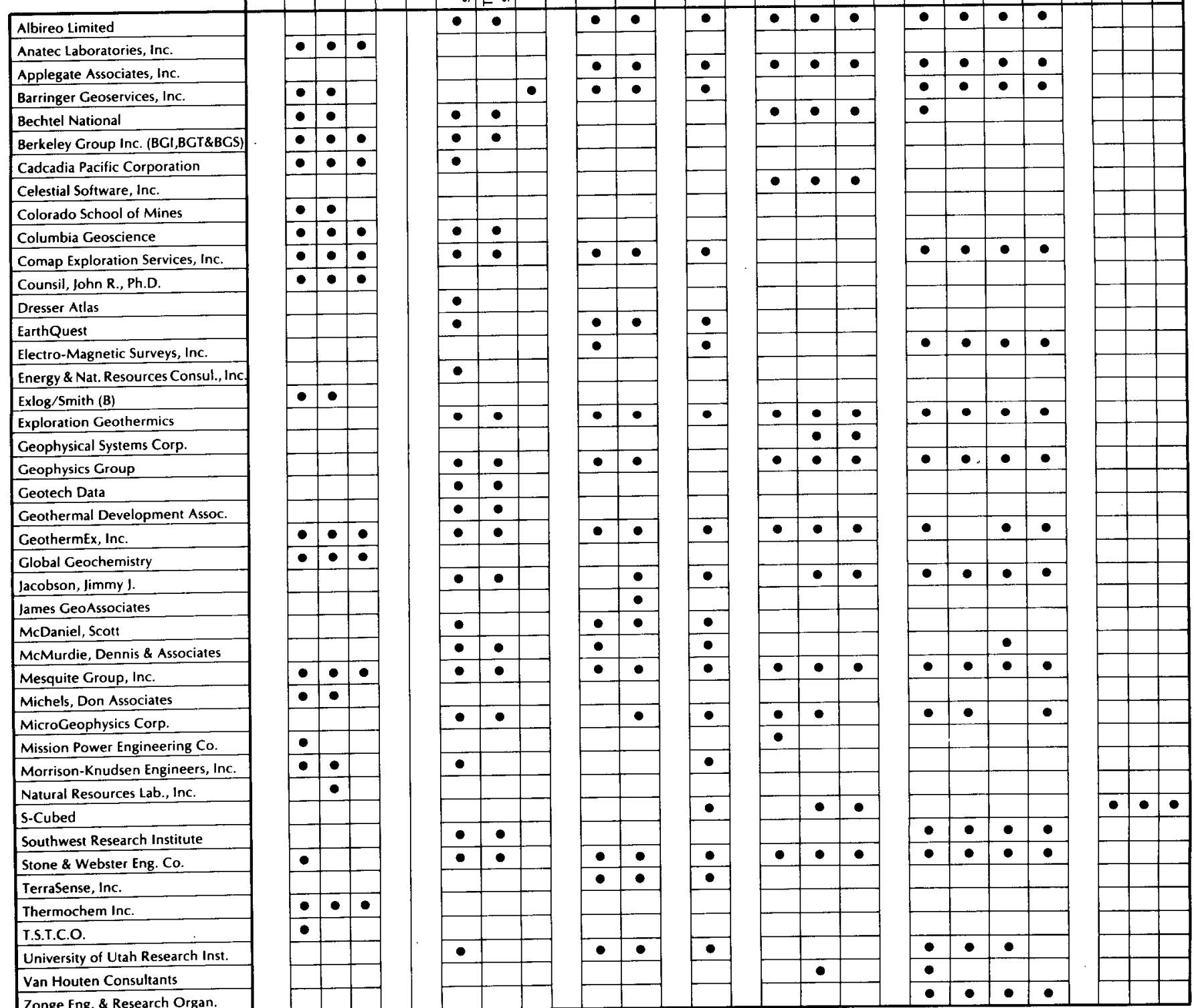




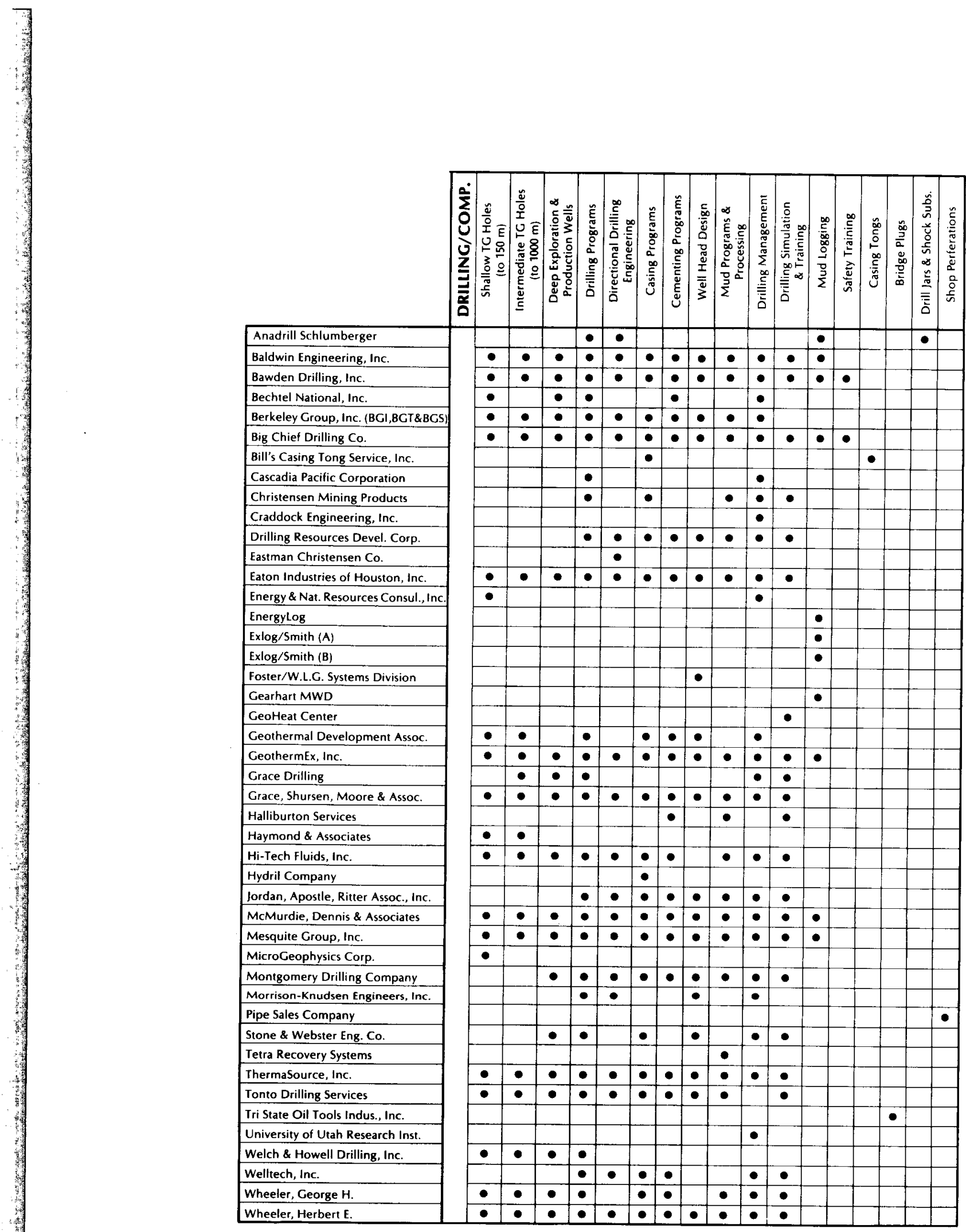


Applegate Associates, Inc.

Baldwin Engineering, Inc.

Bamford, Robert W. \& Assoc., Inc.

Bechtel National, Inc.

Berkeley Group Inc. (BGI,BGT\&BGS)

Cascadia Pacific Corporation

Celestial Software, Inc.

Christensen Mining Products

Comap Exploration Services, Inc.

Counsil, John R., Ph.D.

Dresser Atlas

Eaton Industries of Houston, Inc.

Electro-Magnetic Surveys, Inc.

Energy \& Nat. Resources Consul., Inc.

Exlog/Smith (B)

Exploration Geothermics

Gearhart MWD

GeoHeat Center

Geophysical Research Corp.

Geophysics Group

Geotech Data

Geothermal Development Assoc

GeothermEx, Inc.

Grace, Shursen, Moore \& Assoc.

Haymond \& Associates

Intermountain Tech's., Inc.

Jacobson, Jimmy J.

McMurdie, Dennis \& Associates

Mesquite Group, Inc.

Morrison-Knudsen Engineers, Inc.

Pruett Industries, International

S-Cubed

Southwest Research Institute

Stone \& Webster Eng. Co.

ThermaSource, Inc.

University of Utah Research Inst.

Vice, Dan H. \& Associates

Scientific Drilling International
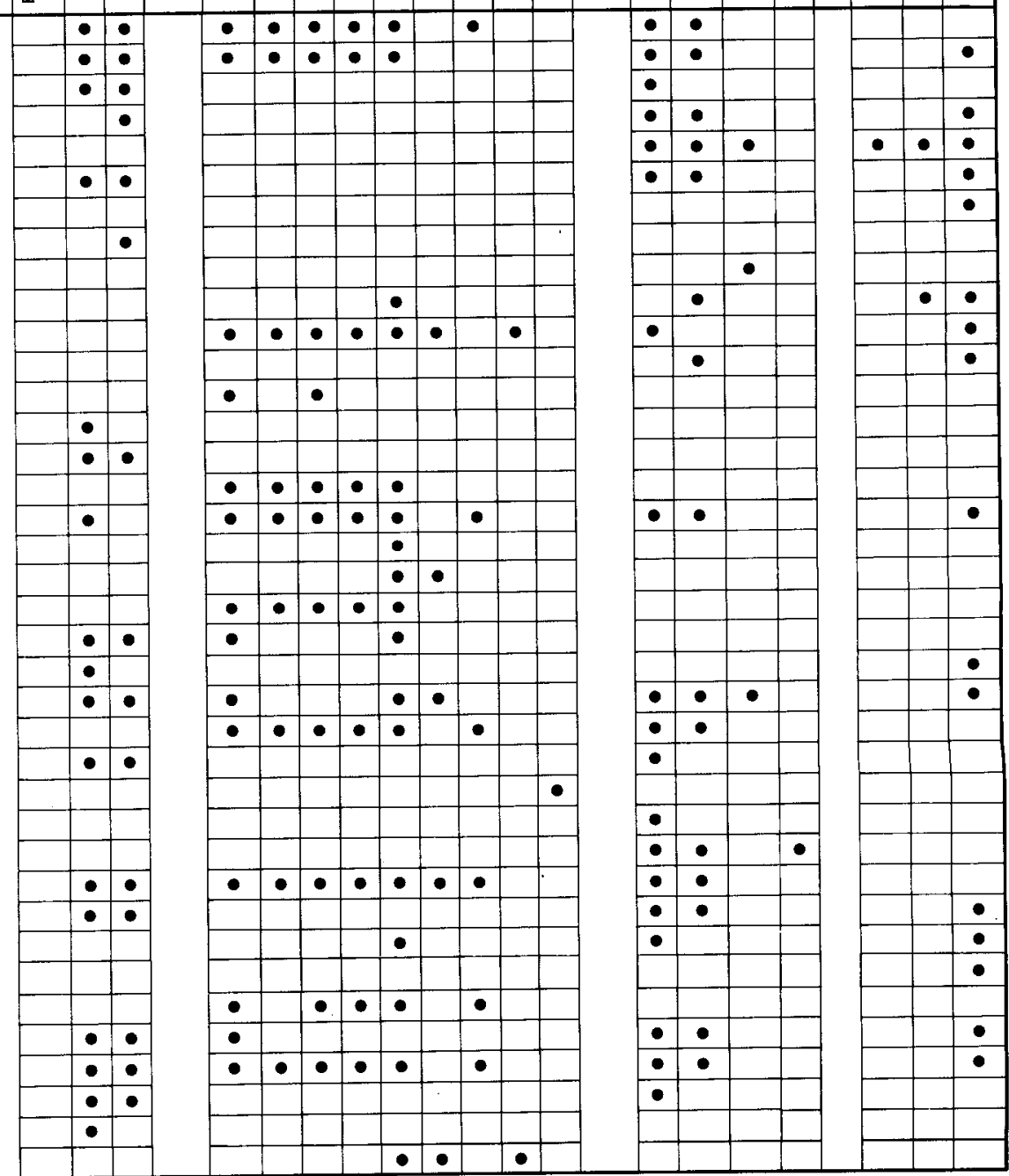


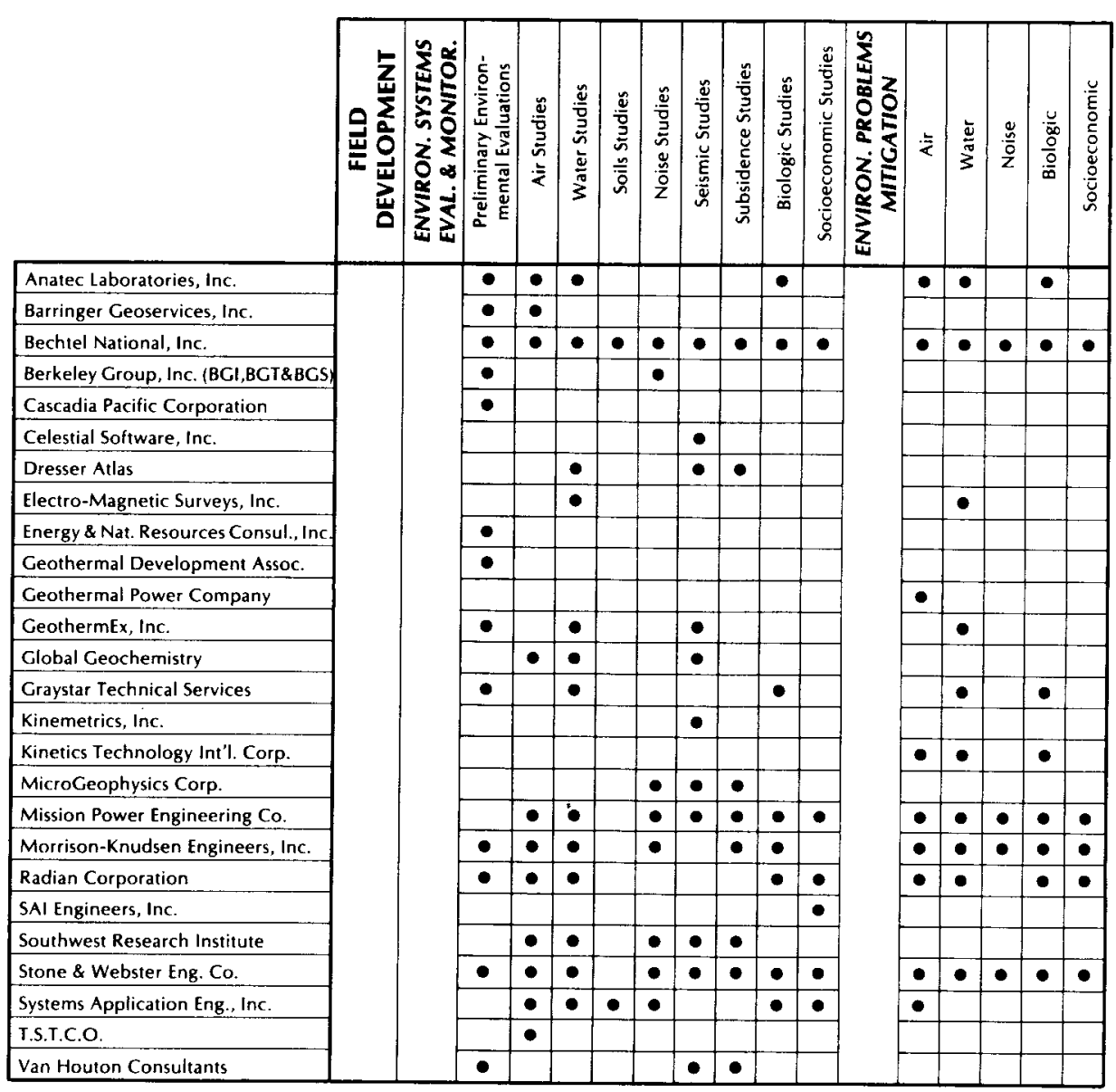




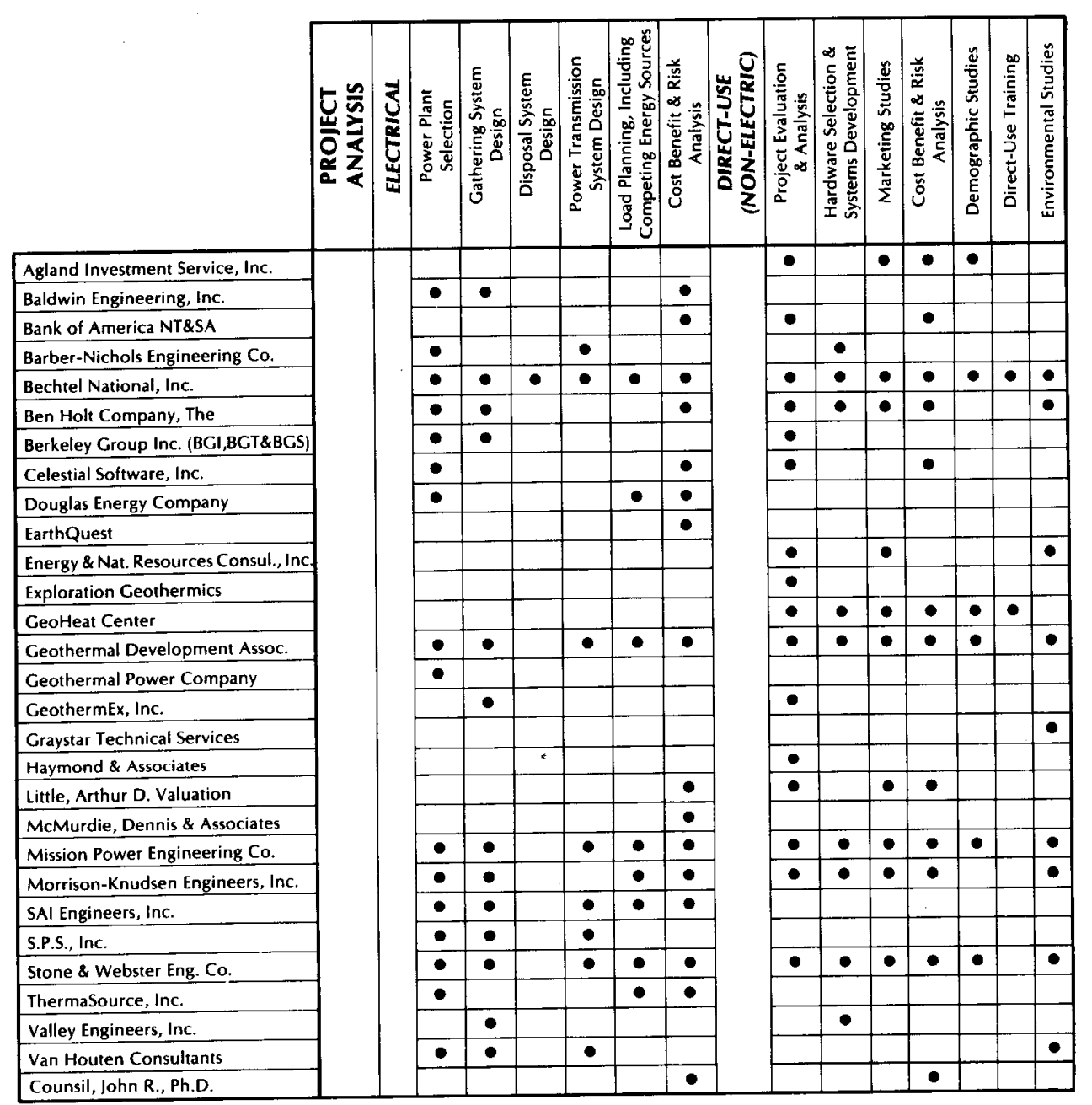




\begin{tabular}{|c|c|c|c|c|c|c|c|c|c|c|c|c|c|c|c|c|c|c|c|c|c|c|c|c|c|c|c|c|c|c|}
\hline 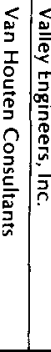 & 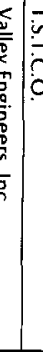 & 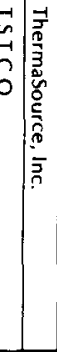 & 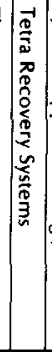 & 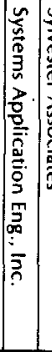 & 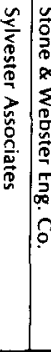 & 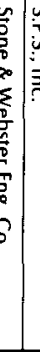 & 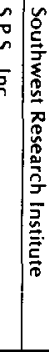 & 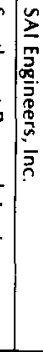 & 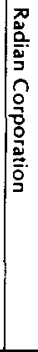 & 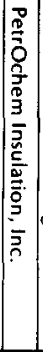 & 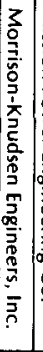 & 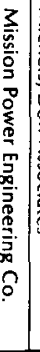 & 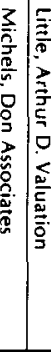 & 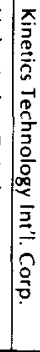 & 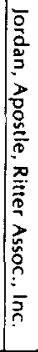 & 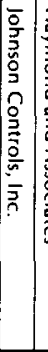 & 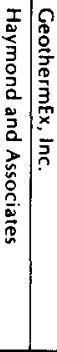 & 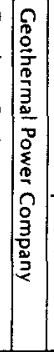 & 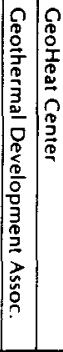 & 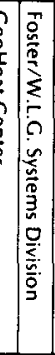 & 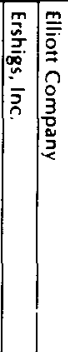 & 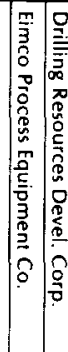 & 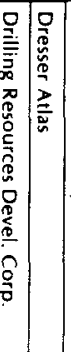 & 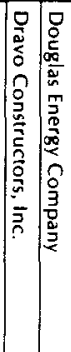 & 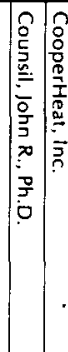 & 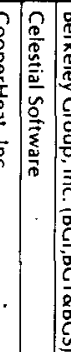 & 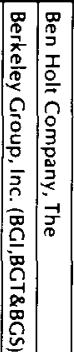 & 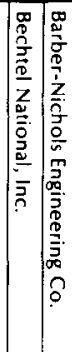 & 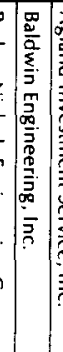 & 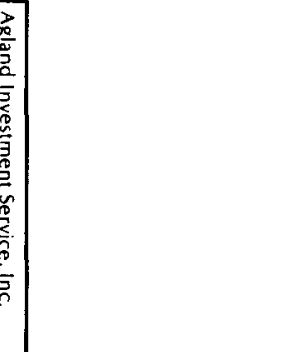 \\
\hline & & & & & & & & & & & & & & & & & & & & & & & & & & & & & & $\begin{array}{c}\text { ENGINEERING } \\
\text { STUDIES \& DESIGN }\end{array}$ \\
\hline & & & & & & & & & & & & & & & & & & & & & & & & & & & & & & ELECTRICAL \\
\hline$\bullet$ & & & & & & $\bullet$ & $\bullet$ & $\bullet$ & & & $\bullet$ & - & & & & & & - & $\bullet$ & & - & & & $\bullet \bullet$ & & $\bullet$ & $\bullet$ & $\bullet \bullet$ & $\bullet$ & Power Plant \\
\hline$\bullet$ & • & & & - & & - & • & $\bullet$ & & & - & $\bullet$ & & & & & $\bullet$ & $\bullet$ & $\bullet$ & - & & & & $\bullet \bullet$ & & - & $\bullet$ & $\bullet \bullet$ & & Gathering Systems \\
\hline & & & & & & & & & & & & & & & & & & & & & & & & & & & & $\bullet$ & & $\begin{array}{c}\text { Fluid Flow Analysis } \\
\text { \& Measurement }\end{array}$ \\
\hline & $-\bullet$ & $\bullet$ & $\bullet$ & & & - & $\bullet$ & $\bullet$ & & & $\bullet$ & $\bullet$ & & $\bullet$ & & & $\bullet$ & $\bullet$ & $\bullet$ & & & $\bullet$ & & $\bullet$ & $\bullet$ & sina & $\bullet \bullet$ & $\bullet$ & $\bullet$ & Waste Disposal Systems \\
\hline & - & & & & $\bullet$ & $\cdot$ & • & $\bullet$ & & & $\bullet$ & $\bullet$ & & & & & & $\bullet$ & $\bullet$ & & & & & $\bullet \bullet$ & & $\bullet$ & - & $\bullet \bullet$ & & Auxiliary Equipment \\
\hline$\bullet$ & & & & & $\bullet$ & - & $\bullet$ & $\bullet$ & & & $\bullet$ & - & & & & & & - & $\bullet$ & & & & & & & $\bullet$ & & $\bullet \bullet$ & & Transmission System \\
\hline & & & & & & & & & & & & & & & & $\bullet$ & & & & & & & & $\bullet$ & & & & & & $\begin{array}{c}\text { Instrumentation \& } \\
\text { Control Systems }\end{array}$ \\
\hline & & & & & & - & - $\bullet$ & $\bullet$ & & & & $\bullet$ & - & & & & & $\bullet$ & $\bullet$ & & & & & $\bullet \bullet$ & & $\bullet$ & $\bullet$ & $\bullet \bullet$ & & $\begin{array}{c}\text { Cooling \& Heat } \\
\text { Rejection Systems }\end{array}$ \\
\hline & - & & & & & & & & & & & 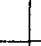 & & & & & & & & & & & & $\bullet$ & & & & $\bullet$ & & General Construction \\
\hline & & & & & $\bullet$ & - & $\bullet$ & $\bullet$ & $\bullet$ & & - & - & - & & & & $\bullet$ & & $\bullet$ & & & & - & $\bullet$ & & - & & $\bullet \bullet$ & $\bullet$ & $\begin{array}{c}\text { Corrosion \& Scaling } \\
\text { Analysis }\end{array}$ \\
\hline & & & $\bullet$ & & & $\bullet$ & & $\bullet$ & & & $\bullet$ & $\bullet$ & - & $\bullet$ & & & • & $\bullet$ & & & & $\bullet$ & & & & & & $\bullet \cdot$ & $\bullet$ & $\begin{array}{c}\text { Chemical Treatment } \\
\text { of Brine }\end{array}$ \\
\hline & & & & & & & & & & & & & & & & & & & & & & & & & & & & $\bullet$ & & Chemical Process Design \\
\hline & & & & & & & & & & & & & & & & & & & & & & & & & & & & $\bullet$ & & Project Monitoring \\
\hline & & - & & $\bullet$ & & - & $\bullet$ & $\bullet$ & & & - & $\bullet$ & $\bullet$ & $\bullet$ & & & $\bullet$ & $\bullet$ & $\bullet$ & & & & & $\bullet \bullet$ & $\bullet$ & - & $\bullet \bullet$ & $\bullet$ & - & Project Management \\
\hline & & & & & & & & & & $\bullet$ & & & & & & & & & & & & & & & & & & $\bullet$ & & Insulation Installment \\
\hline & & & & & & & & & & & & & & & $\bullet$ & & & & & & & & $\bullet$ & & $\bullet$ & & & $\bullet$ & & $\begin{array}{c}\text { Stress Analysis \& } \\
\text { Relief Studies } \\
\end{array}$ \\
\hline & & & & & & & & & & & & & & & $\bullet$ & & & & & & & & - & & & & & & & Finite Element Analysis \\
\hline & & & & & & & & & & & & & & & & & & & & & $\bullet$ & & & & & & & & & $\begin{array}{c}\text { Fiberglass Pipe System } \\
\text { Design } \\
\end{array}$ \\
\hline & & & & & & & & & & & & & & & & & & & & & & & & & & & & & & $\begin{array}{c}\text { DIRECT-USE } \\
\text { (NON-ELECTRIC) } \\
\end{array}$ \\
\hline & & & & & $\bullet \bullet$ & $\bullet$ & $\bullet$ & $\bullet$ & & & • & $\bullet$ & & $\bullet$ & & & $\bullet$ & & $\bullet \bullet$ & & & & & & & - & $\bullet \bullet$ & & & $\begin{array}{c}\text { Project Planning } \\
\text { \& Design }\end{array}$ \\
\hline & $\bullet$ & & & & - 1 & - & $\bullet$ & $\bullet$ & & & $\bullet$ & $\bullet$ & & & & & $\bullet$ & & $\bullet \bullet$ & & & & & & & - & $\bullet$ & & & Gathering Systems \\
\hline & • & & & & - 1. & - & $\bullet$ & $\bullet$ & & & $\bullet$ & $\bullet$ & & $\bullet$ & & & $\bullet$ & & $\bullet \bullet$ & & & & & & $\bullet$ & & $\bullet \bullet$ & & & Disposal Systems \\
\hline & & & & & $\bullet \bullet$ & $\bullet$ & $\bullet$ & • & $\bullet$ & & $\bullet$ & • & - & & & & $\bullet$ & & $\bullet \bullet$ & & & & & & & - & & & & $\begin{array}{c}\text { Corrosion } / \text { Scaling } \\
\text { Studies }\end{array}$ \\
\hline & & & & & $\bullet$ & • & & $\bullet$ & & & - & $\bullet$ & $\bullet$ & & & & • & & & & & & & & & & & & & $\begin{array}{c}\text { Chemical Treatment } \\
\text { of Brine }\end{array}$ \\
\hline & & $\bullet$ & & & $\bullet \cdot$ & - & $\bullet$ & $\bullet$ & $\bullet$ & & $\bullet$ & $\bullet$ & & $\bullet$ & & & $\bullet \bullet$ & & $\bullet$ & & & & & & $\bullet$ & $\bullet$ & 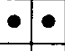 & & & Project Management \\
\hline & & & & & & & & & & & & & & & & & & & & & & & & & & & & & & $\begin{array}{c}\text { Fiberglass Pipe System } \\
\text { Design }\end{array}$ \\
\hline
\end{tabular}




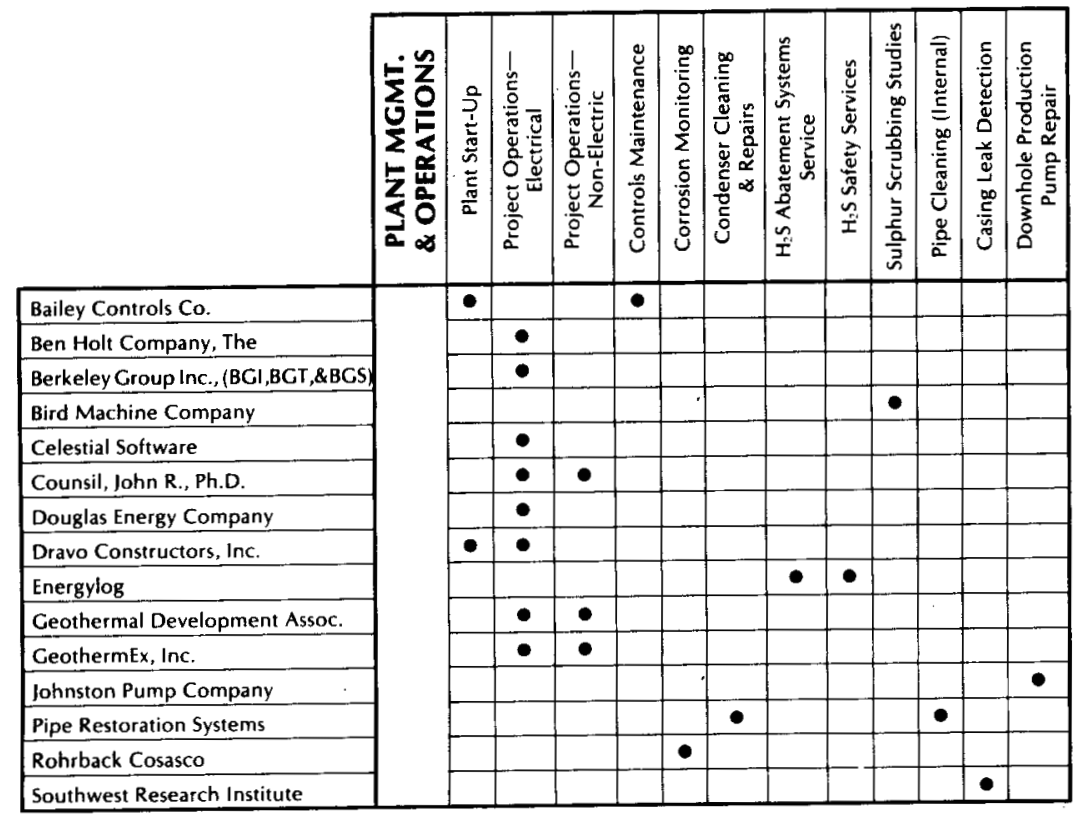
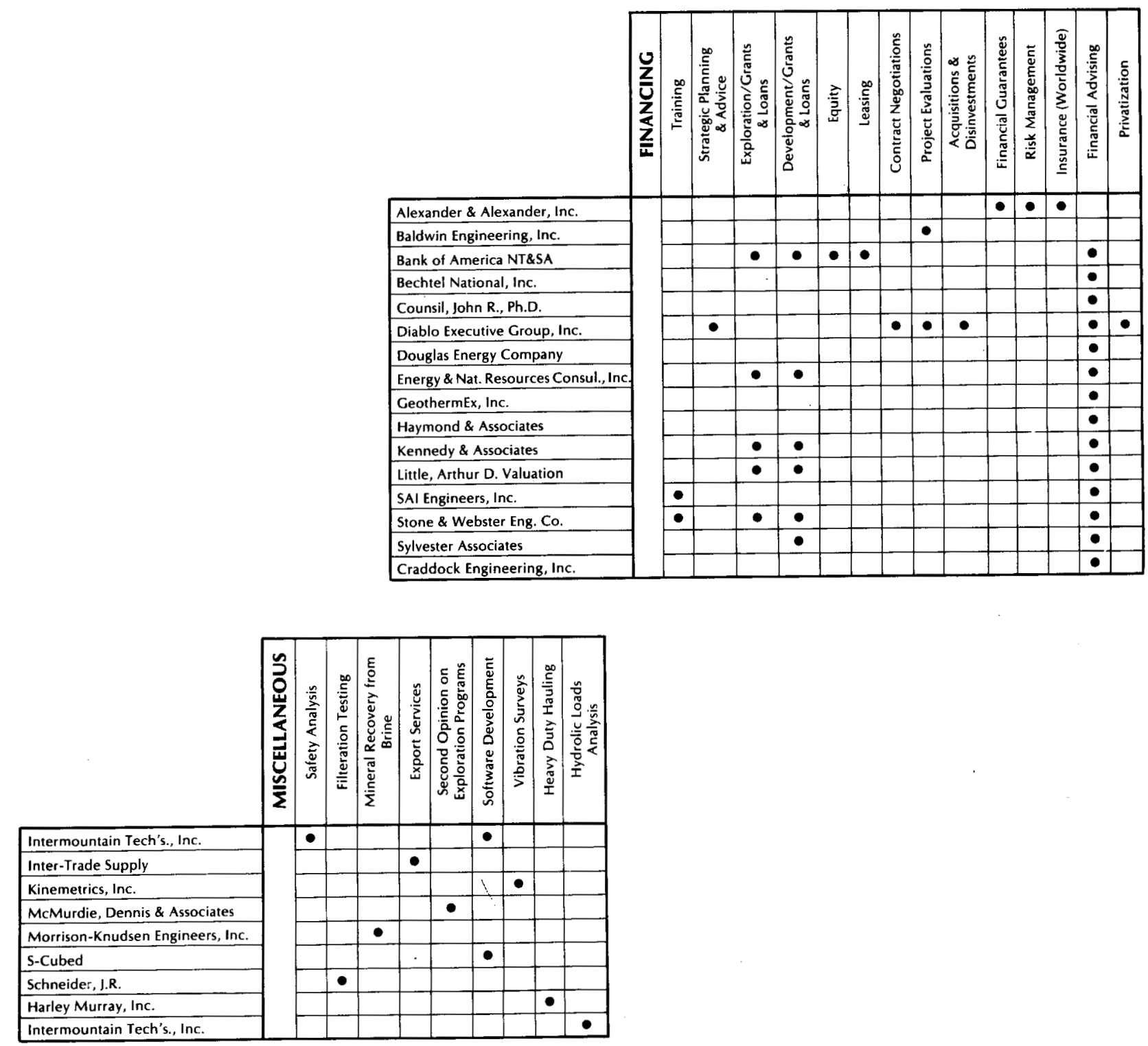